\title{
Marfan's Syndrome with Dissecting Aortic Aneurysm - A Case Report
}

\author{
MOHAMMAD FERDOUS UR RAHAMAN ${ }^{1}$, MOHAMMAD MAINUL HASAN CHOWDHURY ${ }^{1}$, REEHUM HAQUE ${ }^{2}$, \\ NANDITA PAUL ${ }^{1}$, MD. ABU SIDDIQUE ${ }^{3}$, SAJAL KRISHNABANERJEE ${ }^{3,}$ MAHBUBUR RAHMAN $^{1}$ \\ ${ }^{1}$ Department of Internal Medicine, Bangabandhu Sheikh Mujib Medical University (BSMMU), ${ }^{2}$ Department of Medicine, 500 \\ Beded Mugda General Hospital, Mugda, Dhaka, ${ }^{3}$ Department of Cardiology, BSMMU.
}

Address of Corresponding: Dr. Mohammad Ferdous Ur Rahaman, Assistant Professor, Department of Internal Medicine, BSMMU, E-mail: ferdousbsmmu@yahoo.com

\begin{abstract}
Marfan's Syndrome patients with aortic root aneurysm or dissection may present with shortness of breath and chest pain and may be missed during clinical evaluation. Subsequently, sudden aortic rupture may occur in these groups of patients and give rise to fatal outcome. Increasing awareness of Marfanoid signs amongst clinicians are important for early diagnosis and treatment of dissecting aortic aneurysm. Marfan's Syndrome with dissecting aortic aneurysm should not be overlooked among patients with shortness of breath, chest pain and aortic regurgitation. Here we report a case of Marfan's Syndrome who presented with non-exertional chest pain.
\end{abstract}

Key word: Marfan's syndrome, dissecting aortic aneurysm.

\section{Case summary:}

A 32-year-old Bangladeshi male shopkeeper got admitted at BSMMU, Dhaka with occasional chest pain for 1year. Severe central chest pain radiating to back more marked at late night during sleeping associated with arousal from sleep because of chest pain and tightness. Patient also developed dry cough and exersional breathless for same duration. Patient developed erectile dysfunction for 3 month. Patient's father had tall stature and died suddenly because of some unknown disease process. His pulse was $80 / \mathrm{min}$, regular rhythm and good volume. All peripheral pulses were normal without any radio-radial or radiofemoral delay. His blood pressure was 150/20 mmHg with very wide pulse pressure. Neck vein was not engorged. Apex beat was shifted in left $6^{\text {th }}$ intercostals space along anterior axillary line. He had left para-sternal heave with suprasternal pulsation. $1^{\text {st }}$ and $2^{\text {nd }}$ heart sounds were soft and there was presence of early diastolic murmur over the left sternal edge radiating towards the apex. There was also a pistol shot murmur over the left femoral artery. Patient had crepitation over both lung bases without any other abnormality on respiratory system examination. His height was $69.5 \mathrm{~cm}$ and upper segment $(37 \mathrm{~cm})$ was more than lower segment $(32.5 \mathrm{~cm})$. His arm span $(73.5 \mathrm{~cm})$ was also more than height $(69.5 \mathrm{~cm})$. He had positive wrist and thumb sign and had high arched palate (Figure-1). Both eyes were normal on examination. Chest $\mathrm{X}$-ray revealed dilated ascending aorta with cardiomegaly (Figure-2). ECG revealed LVH by voltage criteria. Echocardiography (Figure-3) revealed dissecting aortic aneurysm (false lumen upto arch of the aorta where false lumen re-opens into true lumen), grade-IV AR, and moderate LV systolic dysfunction (EF-45\%). CT-aortogram (Figure-4) revealed aortic aneurysm, largest diameter at root of ascending aorta $(10.5 \mathrm{~cm})$ and smallest diameter at arch of aorta at the site of origin of left subclavian $(5.4 \mathrm{~cm})$. All other part of aorta was normal. Serum TPHA was negative but CRP was raised (45mg/dl). Patient clinically improved with diuretics and âblocker. Urgent consultation from cardiac surgeon was taken and urgent surgery was advised with proper counselling. Finally patient denied taking surgical management considering risk of surgery and cost and got discharge on request.

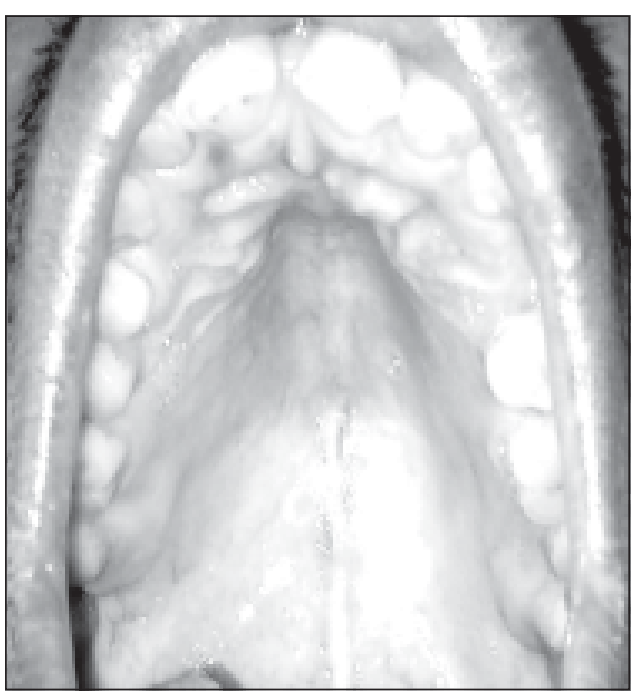

Fig.-1: High arched palate 


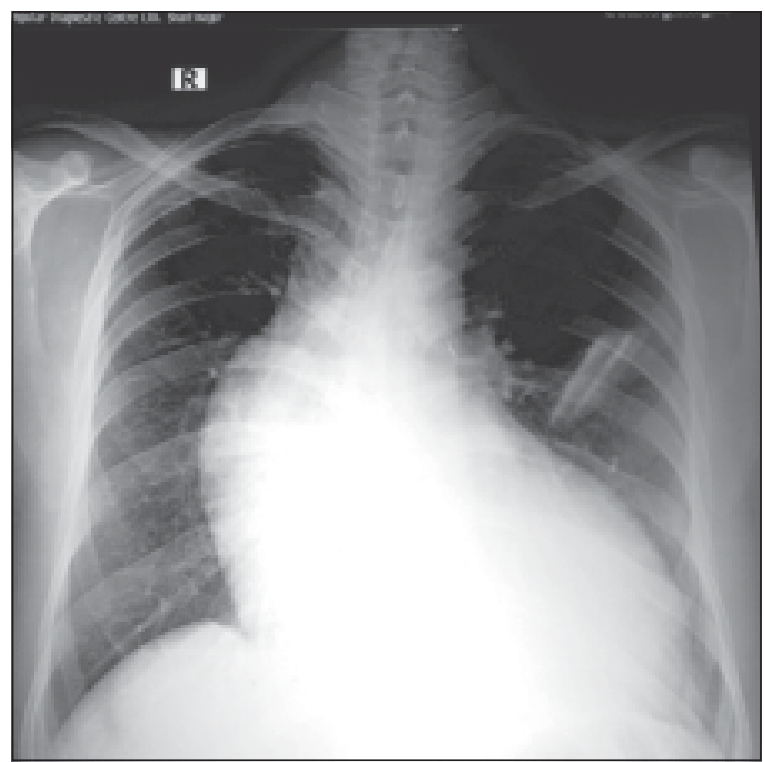

Fig.-2: Chest X-Ray P/A showing dilated ascending aorta with cardiomegaly

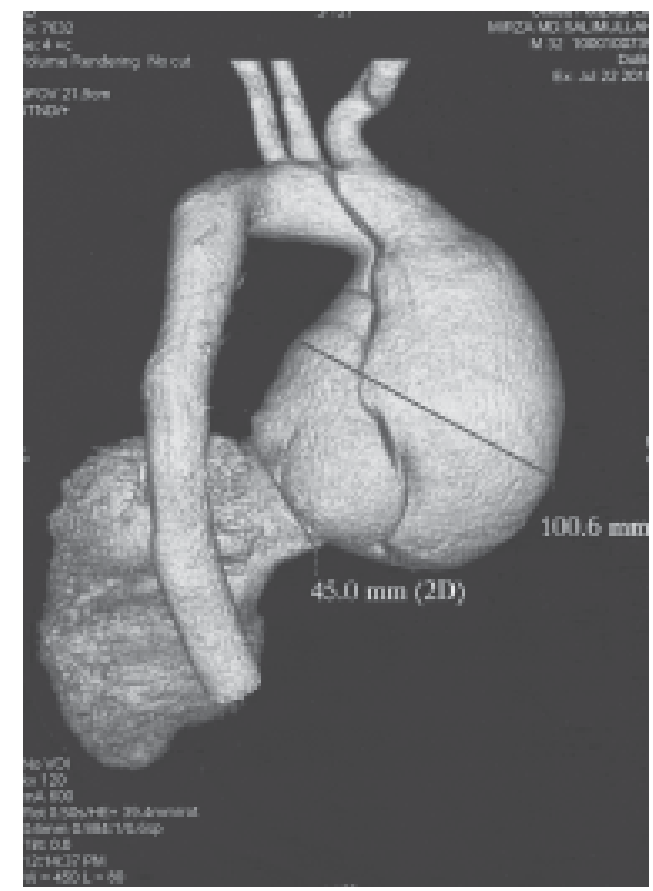

Fig.-4: Aneurysm in ascending aorta

\section{Discussion:}

Marfan's Syndrome is an inherited autosomal dominant connective tissue disorder first described in 1896 by Dr Antoine Marfan ${ }^{1}$. The systems affected in MFS are neurological, skeletal, cardiovascular, ocular and respiratory system. Special attention must be directed to
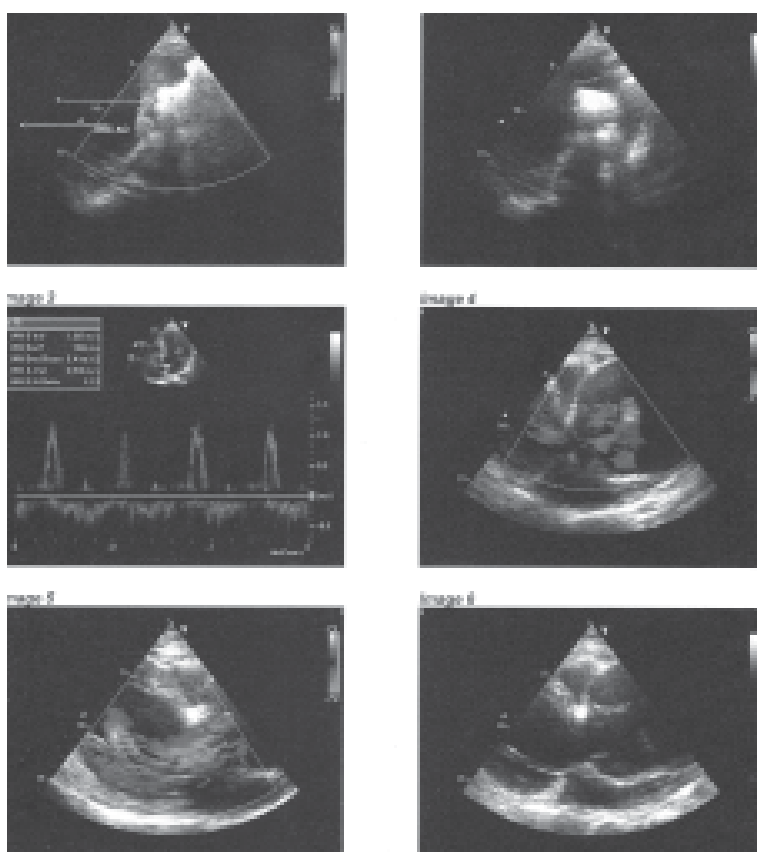

Fig.-3: Dissecting aortic aneurysm

changes in the aortic root, the main cause of mortality. Cystic medial degeneration is seen on histopathological analysis because of elastic fibre fragmentation and paucity of smooth muscle cells with deposition of collagen and mucopolysaccharides between medial cells. Abnormal fibrillin reduces the compliance and distensibility of the aortic wall to luminal ejection forces and associated increased pulse-wave velocity which leads to progressive aortic dilatation and functional aortic regurgitation. Risk of aortic dissection and rupture ensue when the aortic root width exceeds $55 \mathrm{~mm}^{2}$. Secondary consequences include mitral valve dysfunction, left ventricular dilatation, pulmonary artery dilatation, cardiac failure and myocardial infarction. Diagnosis of Marfan's syndrome includes involvement of the skeletal system alongwith involvement of two other systems, with the requirement of at least one major manifestation (ectopia lentis, aortic dilatation/ dissection, or dural ectasia) ${ }^{3}$. Non-invasive imaging remains the method of assessment of the severity of cardiovascular disease aiding timely prophylactic surgical intervention. Plain radiographs may detect mediastinum widening. Trans-oesophageal and Doppler echocardiography can detect aortic aneurysm on the basis of evidence of root dilatation and aortic regurgitation at the sinuses of Valsalva ${ }^{4}$. MRI can detect significant aortic root dilatation by delineating actual-to-predicted aortic root dimension ratios more than 1.5. Though aortography is not $100 \%$ sensitive, it is the main diagnostic tools for 
detection of aortic dissection. Diagnostic gene sequencing can identify an FBN1 mutation within a family but it is an expensive process and not commonly available ${ }^{2}$. The risk of aortic dissection is intimately linked to mortality in MFS patients and there is no definite method to predict the timing of a dissection. Assessment of aortic root widths by measurement at the level of the tips of the valve leaflet may guide decision making for elective aortic root replacement ${ }^{5}$. As a rule, a width of $60 \mathrm{~mm}$ will present a $10 \%$ risk of rupture within the next year, irrespective of site or etiology ${ }^{5}$. Current guidelines advocate prophylactic replacement when the width is $55 \mathrm{~mm}$ in adults; $50 \mathrm{~mm}$ in children. A significant increase in diameter per six-month echocardiography (e.g. 3mm) should be promptly treated without waiting for the diameter to reach $55 \mathrm{~mm}^{6}$. The surgical indications for aortic regurgitation do not alter in a Marfan's syndrome patient. Valve replacement is indicated if there are symptoms attributable to regurgitation and/or evidence of an increase in left ventricular end systolic dysfunction.

\section{Conclusion:}

Marfan's syndrome patients present with shortness of breath due to aortic regurgitation has a high risk of mortality if left undiagnosed. A thorough history, examination and timely investigations among suspected patient with shortness of breath can provide early diagnosis which may save lives.

\section{References:}

1. Younas S, Coupe M, Younas S. Aortic Root Dilatation and Marfan's Syndrome: An Alternative Diagosis. MJM 2005;8(2):128-34.

2. Dean JCS. Management of Marfan Syndrome. Heart 2002;88:97-103.

3. Beighton P, De Paepe A, Danks D, Finidori G, Gedde-Dahl T, Goodman R, et al. International Nosology of Heritable Disorders of Connective Tissue. Am J Med Genet 1988; 29:581-94.

4. Treasure T. Cardiovascular Surgery for Marfan Syndrome. Heart. 2000; 84:674-78.

5. Shores J, Berger KR, Murphy EA, Pyeritz RE. Progression of Aortic Dilatation and the Benefit of Long-term -adrenergic blockade in Marfan's Syndrome. N Engl J Med 1994; 330:1335-41.

6. Treasure T, Reynolds C, Valencia O, Child A, Gallivan S. The timing of aortic root replacement in the Marfan syndrome: computer aided decision support. In: Enker J, editor. Cardiac Surgery and Concomitant Disease. Darmstadt: Springer; 1999. p. 91-8. 